Las estrategias de marketing ecológico en cadenas minoristas, en una perspectiva internacional

\title{
LAS ESTRATEGIAS DE MARKETING ECOLÓGICO EN CADENAS MINORISTAS, EN UNA PERSPECTIVA INTERNACIONAL
}

\section{GREEN MARKETING STRATEGIES IN RETAIL CHAINS: FROM AN INTERNATIONAL PERSPECTIVE}

\author{
James Pulido Novoa* \\ Ernesto Fidel Cantillo Guerrero** \\ Universidad minute de Dios, Sede Costa Norte
}

RECIBIDO: Febrero 2 de 2014

ACEPTADO: abril 12 de 2014

\section{RESUMEN}

El objetivo del presente artículo de investigación consistió en identificar, desde una perspectiva internacional, las distintas estrategias de mercadeo ecológico, aplicadas por un grupo de cadenas comerciales minoristas. La técnica de investigación fue la revisión documental y se utilizó el método analítico. El trabajo se desarrolló en tres etapas: en la primera, se seleccionaron 50 fuentes de información; posteriormente, se construyó un mapeo con los términos claves pertinentes al objeto de estudio; por último, se redactó este artículo. Se estudiaron las estrategias de comunicación dirigidas a los consumidores y clientes, con relación a la oferta de productos y servicios amigables para el medio ambiente. Como resultado de investigación, se determinó que las cadenas minoristas internacionales con sede en Colombia realizan una labor de mercadeo ecológico mediante una estrategia clara de comunicación con los consumidores. Se concluyó que las cadenas comerciales minoristas asumen las tendencias del mercadeo afines a los intereses del Estado colombiano (Plan estratégico nacional de mercados verdes de la república de Colombia), y que dichas cadenas juegan un papel fundamental como intermediarios comerciales de productos y servicios amigables para el medio ambiente.

Palabras clave: Marketing ecológico, producto eco amigable, cadenas minoristas, responsabilidad medioambiental.

\section{Referenciar este articulo (APA)}

Pulido Novoa, James \& E. Cantillo-Guerrero. Las estrategias de marketing ecológico en cadenas minoristas, en una perspectiva internacional, En desarrollo Gerencial, revista de la facultad de ciencias económicas, administrativas y contables de la universidad Simón Bolívar. 6 (1) P.118-143.

\footnotetext{
* Director regional de la costa Norte, Corporación Universitaria Minuto de Dios, jpulido@uniminuto.edu

** Magister en mercadeo, Coordinador de Investigación, Corporación Minuto de Dios , Barranquilla, Ernerto.cantillo@uniminuto.edu 
Las estrategias de marketing ecológico en cadenas minoristas, en una perspectiva internacional

\begin{abstract}
The aim of this research article was to identify different green marketing strategies implemented by a group of retail chains, from an international perspective. A documentary review was the technique used, along with the analytical method. This article was developed in three stages; in the first stage 50 sources of information were selected; thereafter, a mapping was built with the key terms relevant to the subject matter; and finally, the draft article. The strategies focused on the communication to consumers and clients regarding the offer of products and/or amicable services to the environment, were studied. As a result of this research, it was determined that retail chains with headquarters in Colombia carry out green marketing work through a clear communication strategy with consumers. It was concluded that market trends that agree with the interests of the Colombian government (plan estratégico nacional de mercados verdes de la república de Colombia) converge with the commercial retail chains, which play an essential role as commercial intermediaries of products and/or amicable services to the environment.
\end{abstract}

Key words: Green marketing, Eco-amicable product, Commercial Retail Chains, Environmental responsibility. 


\section{INTRODUCCIÓN}

En su obra, Los diez pecados capitales del marketing, Kotler (2004) enuncia los grandes errores en los que incurren las empresas en cuanto a la dirección comercial. Dos de esos pecados se enfocan en el consumidor y en el mercado: el primero de ellos, nos habla sobre las fallas comunes de una empresa que no está lo suficientemente orientada hacia el mercado y dirigida a los consumidores; y el segundo se refiere a las empresas que no logran entender del todo a sus clientes potenciales. Esta falta de orientación hacia el mercado y el consumidor, de las cuales puede adolecer un determinado número de empresarios que olvidan medir las tendencias del mercado objetivo y de realizar la planeación y auditoría de mercadeo acorde con el escenario del consumidor, afecta a la cadena de valor de la empresa en las actividades primarias (Porter, 2002).

$$
\text { De modo que un factor }
$$

determinante para generar valor $\mathrm{y}$ competir es el análisis de las tendencias de consumo, respecto a lo cual, las empresas deben tomar en cuenta a los consumidores que se preocupan por el medio ambiente, pues, justamente representan una importante opción que crece en distintos países del globo. Así, en Alemania y Suecia, el consumo de productos ecológicos crece a una tasa anual de $14 \%$ y $20 \%$, respectivamente. En su mayoría, los consumidores adquieren tales productos tanto en los supermercados tradicionales como en supermercados exclusivos de productos ecológicos (Yáñez, 2009).

Investigaciones hechas en Hong Kong informan también sobre el creciente valor que adquieren los productos verdes en los consumidores jóvenes (Lee, 2008); a lo que se debe 
agregar el mercado de la energía solar fotovoltaica, que creció un $20 \%$ en el 2009 con respecto al 2008 (Solarbuzz, 2009).

Cifras como las anteriores sugieren a las empresas, considerar el factor medioambiental en sus decisiones estratégicas de carácter corporativo y de marketing, dado que el consumidor, preocupado por los recursos naturales y la biodiversidad, desea, y en algunos casos exige, que los productos sean amigables con el medio ambiente (Bravo, 2005).

Ahora bien, uno de los sectores estratégicos de la economía que más contacto tiene con gran parte de los consumidores colombianos es el de los almacenes de cadena, empezando porque a diario, en el caso de hipermercados, reciben la visita de 92.766 empleados quienes, a su vez, son consumidores (DANE, 2010). Este motivo y las cuestiones previamente consideradas son suficientes para el planteamiento de tres preguntas específicas para resolver en el desarrollo de este artículo: ¿Cuáles son los supermercados o hipermercados mundialmente conocidos, que se puedan tomar como muestra de casos exitosos en mercadeo ecológico?, ¿qué compromisos de responsabilidad tienen con el medio ambiente los principales almacenes de cadena ubicados en Colombia?, ¿ofrecen productos o servicios que en su esencia son ecológicos y fabricados a través de procesos con materiales limpios?

\section{MÉTODO}

\section{Diseño}

La metodología utilizada en este trabajo consistió en la revisión de fuentes secundarias (libros, revistas científicas, revistas en Internet, datos estadísticos de agencias) y en análisis de casos, a través de las direcciones electrónicas de 
un determinado grupo de empresas dedicadas al negocio del "retail" o cadenas comerciales minoristas.

\section{Participantes}

Las cadenas minoristas nacionales e internacionales que funcionan en la ciudad de Barranquilla

\section{Instrumentos}

La técnica de investigación utilizada fue la revisión documental

\section{Procedimiento}

El estudio se realizó en tres etapas: la primera comprendió la selección de la fuentes secundarias; en la segunda etapa se realizó el análisis de los hallazgos y, en la última, la redacción del artículo.

\section{RESULTADOS \\ Los hallazgos obtenidos en esta investigación se centraron en el análisis de los casos internacionales y colombianos de estrategias de}

marketing ecológico. A partir de la identificación de las estrategias establecidas, se reconocieron los medios por los cuales este tipo de organizaciones realiza su labor de responsabilidad social empresarial y, a la vez, resulta atractiva para el comprador, al entender éste que las organizaciones donde compran sus productos son eco-amigables y brindan productos ecológicos de calidad.

En específico, se detectaron estrategias ecológicas aplicadas a los siguientes factores: Productos, procesos, recursos físicos, optimización de la cadena de suministro (logística inversa), ubicación y eficiencia energética. Entre los casos exitosos internacionales de estrategias de marketing ecológico en cadenas comerciales minoristas están La corporación IKEA es una organización multinacional sueca, que se dedica a la venta minorista de muebles y objetos para el hogar. Esta ha implementado 
estrategias de gestión ambiental, que hace públicas a través de acciones de mercadeo ecológico como, por ejemplo, el diseño de paquetes planos (fabricados con materiales biodegradables), que facilitan una optimización en la distribución de espacios en el almacén y en el vehículo transportador. Esto garantiza el transporte de un mayor número de unidades por viaje, a la vez que contribuye a la reducción de emisiones anti-ambientales por el menor consumo de combustible. A lo anterior se suma el manejo del código de conducta con los proveedores, a quienes se les exige sistemas de transportes con combustibles limpios (Políticas de reducción de residuos sólidos IKEA Corporation, 2010).De igual forma, IKEA realiza campañas de punto de recogidas de residuos eléctricos para los clientes y promueve el reciclaje de los mismos, intentando con esto reparar los productos en lugar de simplemente desecharlos; de modo que, al final, las piezas se utilizan de recambio $y$ se venden a precios reducidos en zonas de la tienda especialmente designadas.

\section{WALT-MART}

Esta organización trabaja el concepto del mercadeo ecológico desde diferentes puntos de vista. De manera concreta, en su campaña de comunicación de las acciones de mercadeo ecológico, figuran estrategias como: El ahorro de millones de dólares, cambiando bombillas de iluminación incandescente por bombillas CFL (Romero, 2010); la renovación de sus equipos de refrigeración de baja y media temperatura, que eran iluminados con lámparas fluorescentes tradicionales, por refrigeradores con iluminación proporcionada por LEDs, que se activan por movimiento, los cuales, estima la compañía, ahorrarían cerca de US\$2.6 millones en costos de 
energía, y contribuiría a la reducción de emisiones de $\mathrm{CO}^{2}$ en un costo cercanas a 35 millones de libras anuales. Por demás, Walt-Mark se ha propuesto objetivos como lograr que sus nuevas tiendas usen 30\% menos de energía que las actuales, y ha convidado a cerca de 60.000 proveedores a producir bienes que no sean dañinos para el medio ambiente, exigiendo a estos mismos que reduzcan el total del material de empaque en un 5\% para el año 2013 (Romero Castro, 2010).

\section{COSTCO}

Esta empresa utiliza estrategias de mercadeo que incluyen un programa para reducir las emisiones de gases de efecto invernadero, a través de medidas de eficiencia energética. Costco encontró que la electricidad comprada alcanza el 84 \% de su huella de carbono en el 2007. S Su programa de energía pone especial énfasis en diseños de edificios energéticamente eficientes, como ocurre con la instalación de paneles solares fotovoltaicos en las azoteas de los depósitos, comenzado por sus tiendas de California y Hawái. En este último se encuentra el mayor panel solar.

Casos colombianos de marketing ecológico en cadenas comerciales minoritas

\section{Almacenes Éxito divulga acciones ambientales, tales como:}

- www.miplanetaexito.com. Esta Página Web es la encargada de divulgar las acciones ambientales realizadas por la empresa, lo cual es quizás la mejor acción de mercadeo ecológico hecha por un Retail en Colombia. Los colores que identifican la página guardan una relación con los colores institucionales del Grupo Éxito, y en ella se encuentra información actualizada de las actividades que desarrolla la organización para 
proteger el medio ambiente, así como recomendaciones a los consumidores sobre acciones positivas hacia el mismo.

- Publicación de las distintas actividades de ahorro de recursos, a través del informe de sostenibilidad (Almacenes Exito S.A., 2010)

- Los ingresos obtenidos por valor de $\$ 4.950$ millones en la venta de productos orgánicos y ecológicos, representados en 105 referencias de productos que se comercializan después de comprobar que estos cumplen con los correspondientes certificados expedidos por la autoridad competente.

- La consecución de la certificación ISO 14001/04 del ICONTEC en Éxito Bello (departamento de Antioquia) por su acertada gestión ambiental, convirtiéndose así en el primer almacén de cadena de Colombia en lograrlo.
- Es la primera cadena de comercio de cubrimiento nacional que empaca las compras de sus clientes en bolsas plásticas biodegradables y bolsas reutilizables. Su uso representa puntos en la tarjeta de fidelización de sus clientes.

Actualmente las cadenas minoristas, de Almacenes Éxitos, están haciendo esfuerzos, para tener mayor cobertura, han optado por realizar alianzas estratégicas, con las tiendas de barrios del sector sur de Barranquilla, proporcionando a estas toda la logística, para promocionar sus productos de marca propia un día a la semana, desde este escenario también, se proyecta la cultura del marketing ecológicos e igualmente sucede con las tiendas de barrios Express, las cuales han venido fortaleciendo el sector de tiendas de barrios.

$$
\text { De acuerdo a lo expuesto }
$$
anteriormente Peralta (2011): 
Las estrategias de marketing ecológico en cadenas minoristas, en una perspectiva internacional

“Tendero

experto:

Denominamos así a quien tiene

una trayectoria en el negocio, es

el empresario que trata de sacar

ventaja competitiva a su

negocio, con propósitos incluso

de expandir su mercado y

aplicando estrategias de

Marketing uno a uno y de

desarrollo de mercado” P. 174.

Carrefour (actual Jumbo)

En su portal web,

www.carrefour.com.co, sección

de responsabilidad, hace

referencia a su campaña “Bolsas

reutilizables Carrefour”, con la

que comunican la razón de la

actividad e incentivan a los

clientes al uso de las mismas,

mostrándoles que las bolsas

reutilizables son una solución

de largo plazo. $\quad$ Por otra

parte, en Colombia, Carrefour

ha sido pionero en ofrecer bolsas de tela reutilizables en

todos sus hipermercados, con el propósito de que el comprador lleve su mercado en ellas y racionalice su consumo de bolsas plásticas. Esta iniciativa es la primera avalada por el Ministerio de Ambiente, Vivienda y Desarrollo Territorial. Además, la reutilización de una bolsa evita el uso de al menos 15 bolsas de plástico por cada compra.

Por otro lado, en línea con los principios del Pacto Mundial de las Naciones Unidas en cuanto al respeto del medio ambiente, las estrategias ambientales de Carrefour comprenden:

- $\quad$ Reducción del impacto ambiental en sus tiendas.

- $\quad$ El desarrollo de productos que respeten el medio ambiente y la biodiversidad. 
- $\quad$ La reducción del 30\% de consumo de energía del grupo

Carrefour al año 2020.

- $\quad$ La sensibilidad y educación de los consumidores y de sus colaboradores sobre el consumo responsable y el desarrollo sostenible.

- $\quad$ Home Center $\quad$ En su página Web, esta organización presenta un pop-up dinámico, en el cual enseña la bolsa reutilizable con frases ecológicas y emotivas que incentivan su uso. Adicionalmente, Home Center maneja una línea amplia de productos amigables para el medio ambiente, así como de productos fabricados por empresarios comprometidos con planes de gestión ambiental. Todo esto se comprueba cuando uno ingresa al portal Web de la empresa (www.homecenter.com.co), sección hogar verde, donde se encuentra una amplia gama de productos comprometidos con el ahorro de recursos (grifería, bombillos de baja potencia, canecas para clasificación de basuras, lámparas solares, pinturas ecológicas, sellos para aislamiento térmico, entre otros). Otro detalle para resaltar en la Web de esta empresa es la campaña de acciones para el hogar. Mediante esta, la empresa no solo promueve la compra de los productos que comercializa, sino que también ofrece información valiosa a los consumidores y clientes sobre el ahorro de recursos relacionado con cada uno de los bienes de sus hogares, como son: electrodomésticos, muebles de sala, comedor, regaderas y otros. Finalmente, en la divulgación de su programa de responsabilidad social empresarial, Home Center incluye al medio ambiente, y precisa esto 
como una responsabilidad de toda la compañía, que contribuye a preservarlo desde una óptica que involucra las operaciones totales de la misma.

\section{Almacenes Olímpica}

La única acción ambiental que esta organización divulga es la adopción del elefante de la Fundación Zoológico de Barranquilla. A pesar de que en sus puntos de ventas manejan líneas de productos orgánicos y ecológicos, esta cadena de almacenes no saca provecho de esta actividad para realizar más acciones de mercadeo ecológico.

\section{Marketing supermercados especializadas ecológico en y tiendas}

Cifras de la muestra mensual de comercio al por menor, elaborada por el DANE para el mes de septiembre del 2010, muestran un crecimiento en $18,6 \%$ de las ventas del comercio minorista con respecto al mes de septiembre del año anterior (DANE, 2010). Tales estadísticas incluyen a los hipermercados y demás almacenes de cadena, que para este articulo denominamos supermercados y tiendas especializadas. $\quad \mathrm{Al}$ observar las direcciones electrónicas de establecimientos como FEDCO, SURTIFRUVER de la Sabana, Almacenes YEP y La 14, se encontró que ninguno de ellos realiza acciones de mercadeo ecológico, debido a la falta de divulgación de los programas ambientales llevadas a cabo en sus organizaciones. Estas organizaciones tampoco promueven explícitamente productos o servicios ecológicos, aunque de forma indirecta podría considerarse como tal el enfoque de SURTIFRUVER de la Sabana, tienda especializada en la venta de frutas y verduras, que, en su página 
web, promueve el consumo de frutas y verduras por salud y bienestar.

Las empresas en cuestión

asumen compromisos

de responsabilidad medio ambiental para el cumplimiento de las estrategias anteriormente expuestas, y formulan políticas con base en la responsabilidad social $\mathrm{y}$ en un enfoque ecológico. En general, las políticas de estas organizaciones consisten en mantener una cadena de valor sostenible en el sistema de actividades del negocio. Para esto, se organizan desde el inicio hasta el final de todo el ciclo de vida del producto, generando un valor agregado para todos los funcionarios y clientes de la organización, quienes aseguran así un éxito perdurable contribuyendo de esta manera al bienestar de la humanidad y del medio ambiente.

Se logró identificar que las organizaciones objeto de estudio poseen en su oferta productos que reúnen la condición de ser amigable para el medio ambiente.

Teniendo en cuenta variables como volumen de ventas (BPR Benchmark, 2010) y presencia en el territorio nacional, las cadenas comerciales minoristas ubicadas en Colombia se clasifican en dos categorías: 1) hipermercados, y 2) supermercados $\quad y \quad$ tiendas especializadas. En este sentido, es importante aclarar que el presente estudio se limita a supermercados y tiendas especializadas, que manejan consumo masivo relacionados con la canasta familiar, el cuidado y la belleza, descartando otra serie de productos que se comercializan al por menor a través del formato de tiendas especializadas.

En las estrategias de marketing ecológico de este modelo de negocios, se detectó claramente el compromiso de los propietarios de las cadenas y los consumidores con el medio ambiente. 
A continuación, se detallan también aspectos relevantes sobre el particular en las organizaciones:

- Las cadenas minoristas, al igual que otros sectores empresariales, han tomado la iniciativa de aplicar estrategias de mercadeo ecológico, entendiendo que esto no solo constituye un motivo extra de generación de ventas, sino que también muestra a la comunidad el compromiso social $\mathrm{y}$ ambiental que tienen con el entorno, base indispensable para la perdurabilidad de la empresa en un medio cada vez más competitivo. Como ejemplos, podemos citar a las cadenas Wal-Mart, IKEA y Costco. - Los hipermercados colombianos (de capital nacional y extranjero) no están muy distantes de estas iniciativas en estrategias de marketing ecológico, y cuentan con campañas exitosas como la de

\author{
Almacenes Éxito, estrategias de \\ comunicaciones novedosas y \\ atractivas como la de Home Center, \\ y la fuerte promoción de las bolsas \\ reutilizables de Carrefour.
}

A los supermercados y otras tiendas especializadas aún les falta camino por recorrer en cuanto a acciones de marketing ecológico, que les permitan ganar un espacio en la mente de los consumidores ecológicos. Esto no significa que no estén a tiempo de incluir este nicho de consumidores.

Es importante destacar la labor realizada por el Grupo Éxito, principal almacén de cadena en Colombia. La estrategia de marketing ecológico realizada por esta organización constituye un modelo a seguir para otras empresas, al reflejar en sus acciones un fuerte compromiso que se manifiesta en la publicación de 
resultados de su gestión, recalentamiento global, la tecnología, el información vigilada por la firma de auditoría Deloitte. uso de bolsas plásticas, etc. Todo lo cual obliga a las empresas a ofertar

\section{DISCUSIÓN}

\section{Según la Asociación Americana}

de Marketing (AMA, por sus siglas en inglés), el marketing ecológico es el esfuerzo que hace una empresa para producir, promover, empacar y recuperar los productos con un enfoque de responsabilidad y sensibilidad ecológica (Samper, 2009). $\quad$ Por su parte, Polonsky (1997) lo define como un conjunto de actividades diseñadas para generar y facilitar cualquier intercambio de bienes y servicios que permitan satisfacer las necesidades del ser humano, a través del cuidado y protección del medio ambiente. $\quad$ El marketing ecológico implica, entonces, el desarrollo de actividades de preservación de un entorno ambiental, que cada día corre más peligro de autodestrucción, por problemas como el productos $\mathrm{y}$ servicios que no incrementen ese riesgo y, en lo posible, contribuyan a erradicarlo. Así las cosas, cuando una organización decide ofrecer productos o servicios amigables para el medio ambiente, suele utilizar estrategias de comunicación dirigidas a los consumidores que evidencien sus prácticas de mercadeo que se hallan comprometidas con el cuidado del mismo. De esta manera, las distintas variables generadoras de valor en las empresas se transforman, gracias a una gestión ambiental sostenible en la cadena, que nunca pierde la visión de apuntar a un consumidor ecológico. El diseño de productos, el empaque, el embalaje y la publicidad de las líneas de producción, así como el aprovisionamiento, los canales de distribución y las demás áreas de la 
organización han de involucrarse en la estrategia de mercadeo ecológico.

Desde principio de los años 80, el marketing ecológico se convirtió en una tendencia fuerte de aplicación comercial en el mundo, llegándose a considerar la década del 90 como la revolución verde. Sin embargo, durante esas décadas, se vieron muchas campañas y productos relacionados que no obtuvieron los resultados esperados (Vazifehdust, Javad \& Gharib, 2011). Aun así, es notable que, en las normas y en el comportamiento de las personas, se ha producido un cambio en sus actitudes hacia un estilo de vida verde, y es claro que la gente intenta activamente reducir el impacto sobre el medio ambiente (Cherian \& Jacob, 2012). Estos cambios conllevan a que las empresas realicen distintas acciones a su interior para implementar las estrategias de mercadeo ecológico, más aún si el sector de la organización se relaciona con actividades que implican un alto impacto ambiental.

\section{Razones para utilizar el marketing ecológico \\ Como se viene exponiendo, al} tratar de satisfacer las necesidades de los consumidores con conciencia ecológica, recientemente las empresas han decidido desarrollar procesos de producción que contribuyan al desarrollo sustentable de la economía y la sociedad (Salgado y Beltrán, 2009). En este marco, cobra valor el interés por preservar los recursos naturales limitados, el uso eficiente de los mismos, la reducción o reutilización de los residuos, y demás acciones que se enfocan a satisfacer una demanda que hoy exige hechos concretos, pero sin que la empresa pierda su interés básico para la perdurabilidad, la optimización de sus recursos y el logro de objetivos económicos. En este ciclo de satisfacción-optimización y consecución de objetivos, el mercadeo ecológico 
pasa a ser una filosofía comercial relevante, por medio de la cual la empresa aprovecha la gestión ambiental y el uso eficiente de recursos, realizada al interior de ella para divulgarla, bajo los métodos del mercadeo, al entorno y a los consumidores en general.

Ya se ha advertido que, en algunos sectores, el mercadeo ecológico precisa un estricto cumplimiento (por ejemplo, en la minería); pero, en otros, es una oportunidad para proponer nuevos productos y explorar nuevos mercados (ejemplo, agricultura ecológica); mientras que en otras empresas constituye la única forma de ofertar sus productos (ejemplo, energías limpias). Entonces, se puede decir que las organizaciones tienen variadas razones para implementar el mercadeo ecológico, pero todas son en sí oportunidades que permiten a las mismas obtener ventajas competitivas, concepto entendido como la destreza o habilidad especial que logra desarrollar la empresa y que la ubica en una posición de preferencia a los ojos del mercado (Mapcal, 1997).

Fernández (2009) se refiere a otro aspecto de gran relevancia en este tema: la responsabilidad social corporativa, dado que las empresas deben reinvertir voluntariamente para contribuir al mejoramiento del ambiente interno $\mathrm{y}$ social, interactuando con los grupos de interés como clientes, proveedores, empleados, consumidores, y la comunidad general. Actualmente, la responsabilidad social empresarial cumple para la sociedad unos lineamientos, que deben reflejarse en los planes y políticas organizacionales, los cuales deben incluir la dimensión ambiental (Aparicio, 2009). Además, los Estados empiezan a establecer normas y políticas que fomentan y exigen a las organizaciones cumplir con los requisitos en materia 
medioambiental. Para esto, se puede citar, en Colombia, la Ley 1333 del 2009, que establece el procedimiento sancionatorio ambiental. Por último, se debe reconocer la relación costobeneficio a favor de la empresa en esta materia. $\quad \mathrm{Al}$ respecto, el estudio de Aberdeen Group (2008) resalta que un 9\% de los consumidores está dispuesto a pagar un precio superior por los productos ecológicos. Y es que los consumidores de productos ecológicos van entendiendo que estos productos poseen valor agregado, y para el empresario estos productos constituyen, hoy por hoy, una oportunidad valiosa, mediante la cual pueden obtener una rentabilidad por unidad más alta que con los productos tradicionales.

En el proceso de iniciar una estrategia de marketing ecológico en una cadena comercial minorista, el primer paso sugerido es realizar el diagnóstico ambiental de la empresa, y establecer si la empresa se encuentra preparada (cultura-recursosdisponibilidad) para asumir el reto. Si el diagnóstico brinda la tranquilidad suficiente para implementar una estrategia de mercadeo ecológico, la cual debe ir sincronizada con las distintas estrategias establecidas en el plan de marketing, a su vez, el plan de acción de la estrategia de mercadeo ecológico debe contar con una serie de acciones dirigidas a la obtención de mayores ingresos para afrontar el cambio cultural y de hábitos de consumo que han surgido en los consumidores del siglo XXI. Si resumiéramos todo esto en un formato de ecuación, el modelo a proponer sería:

Figura 1. Ecuación de incrementos de ventas en mercados ecológicos

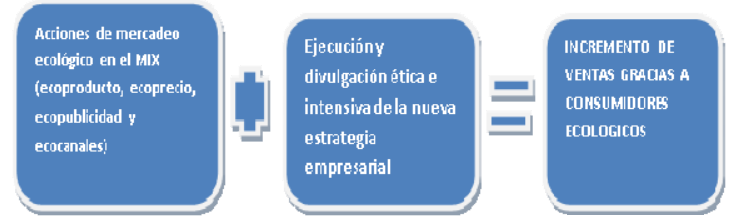

Fuente: Elaboración de autores. 
Acciones ecológicas en

el marketing MIX

El marketing MIX es el eco-producto en sí y la pregunta que debe plantearse al respecto es: ¿Cómo podemos convertir una cadena minorista, que es un canal de distribución, en un ecoproducto? El análisis de casos permite sugerir la hipótesis de que más que un eco-producto, la organización debe construir un eco-marca, es decir, una marca que se logre posicionar como ambientalmente responsable en la mente de un consumidor, tal como lo hacen los hipermercados aquí reseñados.

Otra opción para estas organizaciones es el eco-precio, ya que el retail, sector empresarial que compite ampliamente con la variable precio, exige realizar acciones que optimicen el costo en los procesos, y que a su vez no incrementen la estructura de precio al público.

A través de la eco-publicidad, la organización ha de promulgar las diferentes actividades desarrolladas a favor del eco-marketing, tales como: la reducción de consumos de recursos (energéticos, agua, gas), la organización de brigadas de reciclaje en el barrio o zona urbana en que se encuentra ubicado, y el uso de las redes sociales.

Por último, se recomienda a las organizaciones utilizar los eco-canales: La tienda, el supermercado, son canales de distribución, que como tales pueden establecer acciones ecológicas en el punto de venta (canecas para reciclaje), manejo efectivo de envases retornables, adecuación eco-eficiente en las bodegas de almacenamiento, entre otras. Además, pueden exigir al mayorista demostraciones de responsabilidad ambiental y, así, fomentarlo en los demás actores de la cadena.

\section{Al consistir la estrategia en} migrar de una planeación de mercadeo tradicional a otra de mercadeo ecológico, se requiere que cada uno de 
Las estrategias de marketing ecológico en cadenas minoristas, en una perspectiva internacional

los miembros que conforman la empresa esté $100 \%$ comprometido con ella, esto es, con la intención de divulgar a los clientes y consumidores en general las acciones que la empresa lleva a cabo en pro del medio ambiente y actuar acorde con la planeación de mercadeo ecológica. Con otras palabras, que la organización en su totalidad asuma el gran reto de ser y parecer.

\section{REFERENCIAS}

Aberdeen group. Going green: When "making a difference" aligns with market demand. Recuperado de http://www.marketingpower.co m/ResourceLibrary/Documents/ Content\%20Partner\%20Docume nts/Aberdeen\%20Research/Goin g_Green.pdf Agricultura orgánica o ecológica. Fundación Manuel Mejía y Ministerio de agricultura y desarrollo rural. http://www.minagricultura.gov.c o/archivos/guia_de_agricultura_ ecologica.pdf

Allen, T (2007). Green-marketing, could green marketing be a sustainable competitive advantage for retailers within South Africa? (Tesis de maestría). University of Pretoria, Pretoria.

Almacenes Éxito. (2010). Políticas empresariales de las empresas, estudio de campo técnicas de encuestas.

Amine, L. Smith, J. Challenges to modern consumer segmentation in a changing world: the need for a second step. Multinacional Bussines review. 17(3), 71-99.

Aparicio, J., Valdés, B. Sobre el concepto de responsabilidad social de las empresas. Un análisis europeo comparado. Cuadernos de relaciones laborales, 27, 53-75. 
Bastidas, L. (2009). Sostenibilidad, la tendencia de 2010. Recuperado de www.larepublica.com.co

“Best supermarket: mark \& Spencer”. Abril de 2010. Disponible en www.indepedent.co.uk

Bollana, K (2007). Eco diseño y sustentabilidad, metodología de diseño eco eficiente $y$ sustentabilidad de productos $y$ actividades ecológicas. Trabajo presentado a la Cátedra Galán FAD Universidad de Buenos Aires. Recuperado en http://catedragalan.investigacion accion.com.ar/trabajos/64b8dffc 08d24c9658b07fdf581f006f_eco diseno_y_sustentabilidad.pdf.

Bravo, R., Fraj, E. y Martínez, E. La importancia del factor medioambiental en las estrategias corporativa de marketing: una aplicación al sector de bienes de consumo. Cuaderno de estudios empresariales, 15, 199-224.
Calomarde, J. (1992). Influencia de los factores ecológicos en la decisión de compra de bienes. Recuperado en http://dialnet.unirioja.es/servlet/ articulo?codigo $=2867206$

Calomarde J. (2005, septiembre). Marketing medioambiental. V jornadas técnicas sobre reciclado de aparatos eléctricos y electrónicos. Universidad de Cádiz - España. Recuperado de http://www2.uca.es/grupinvest/cit/Jornadas05_archivos/J ose_V_Calomarde_W.pdf

Camargo, L. (2009). Supermercados ecológicos, tiendas especializadas en productos orgánicos. Recuperado de http://www.elespectador.com/im preso/vivir/articuloimpreso1103 71-supermercados-ecologicos

Chamorro, A (2001): El marketing ecológico. Recuperado de www.5campus.org/leccion/ecom arketing 
Chamorro, A., Miranda F.J., Rubio L. S (2006). El estado de la investigación sobre marketing ecológico en España: análisis de revistas españolas 1993 - 2003. Investigaciones europeas de Dirección y Economía de Empresa. 12(2), 137-156.

Chamorro, A., Miranda, F.J., Rubio L. S. La investigación académica en marketing ecológico: diferencias entre las publicaciones españolas y las internacionales. Recuperado de:http://educamarketing.unex.e s/asignaturas/curso\%20doctorad o/Investigaci\%C3\%B3n\%20en \%20Marketing\%20Ecol\%C3\%B 3gico\%20_Profesores\%20Mk.p $\mathrm{df}$

Chamorro A. y González O.R. (2005). Marketing de productos ecológicos. En Aplicaciones de marketing 93 - 122. Bajadoz: abecedario.
Cherian, Jacob. Jacob, Jolly. (2012). Green marketing: a study of consumers' attitude towards environmental friendly products. Asían social science, 8 (12), $117-126$.

Costco wholesale today. (2010, Abril). Responsibility resonates. Recuperado de http://stacibarsness.com/Docum ents/ResponsibilityResonates_A pril2010.pdf

Departamento Administrativo Nacional de Estadística-DANE, Boletín de Prensa de la Muestra mensual de comercio al por menor. Julio 2010 http://www.dane.gov.co/files/in vestigaciones/boletines/mmcm/b ol_mmcm_jul10.pdf

Di Benedetti, Bill. Costco Dives into product sustainability.Octubre 9 de 2009. www.triplepundit.com 
Ecología verde (2010, enero 4). El auge de los supermercados ecológicos. De David. Recuperado de http://www.ecologiaverde.com/e l-auge-de-los-supermercadosecologicos/

Educa marketing (2005). Los supermercados ecológicos en España. Recuperado de http://educamarketing.unex.es/D ocs/casos/supermercados\%20ec ol\%C3\%B3gicos.pdf

Fernández García, R. (2009). Responsabilidad social corporativa. Editorial Club Universitario. España http://books.google.com.co/boo ks?id=295vqLhaTioC\&printsec $=$ frontcover \&dq=concepto + resp onsabilidad+social+empresarial \&hl=es\&sa=X\&ei=741UU5mc KKatsASW84CYCA\&ved=0C DMQ6AEwAQ\#v=onepage\&q= concepto\%20responsabilidad\%2 0social\%20empresarial\&f=false
Fiegerman, S. (2010, octubre 7). FTC cracks down on Green Marketing. Recuperado de http://www.mainstreet.com/artic le/small-business/marketing/ftccracks-down-green-marketing.

Gerpott, T., Mahmudova, I. (2010). Determinants of Price mark-up tolerance for green electricity lessons for environmental marketing strategies form study of residential electricity customer in Germany. Business strategy and the environment. 19(5), 304. 
IEG LLC. (2009, Septiembre). Turning green into green: taking advantage of eco-friendly sponsorships. Recuperado de http://www.marketingpower.co m/ResourceLibrary/Documents/ Content\%20Partner\%20Docume nts/IEG/2010/turning_green_int o_green.pdf

IKEA Corporation, 2010- Políticas empresariales de las empresas, estudio de campo técnicas de encuestas.

Kotler, Philip. (2004). Los 10 pecados capitales del marketing: indicios y soluciones. Barcelona: Gestión 2000

Lee, Kaman. Opportunities for green marketing: Young consumers. Marketing Intelligence \& planning, 26, 573-586.

McCafferty, D. (2009, febrero) Green washing: don't be misleading by pone "green" marketing.
Recuperado de http://www.lowesforpros.com/gr eenwa

Mapcal, S. A. (1997). La ventaja competitiva. España: Días de santos. See more at See more at: http://brd.unid.edu.mx/entrevista -a-jaime-lopez-francosfundador-y-director-general-deymedia/\#sthash.hnLn8pw8.dpuf

Shing-don't-be-mislead-by-phonygreen-marketing

Olamendi, G. Marketing ecológico. Disponible en www.estoesmarketing.com

Orange, Erica. (2010). From ecofriendly to eco-intelligent. The futurist. Septiembre-octubre, 2832.

Peralta P. (211), Modelo de negocios de tienda de barrio y la brecha tecnológica frente a la competitividad. En Desarrollo Gerencial, revista de la facultad de ciencias económicas, administrativas y 
Las estrategias de marketing ecológico en cadenas minoristas, en una perspectiva internacional

contables. $\quad 3(2), \quad$ 166-187. file://C:/Users/PROFESORES/

Downloads/55-100-1-

PB\%20(6).pdf
Montillaud, Solange. Parasnis, Mandar. (2007). Tiendas verdes y grandes ahorros. Recuperado de http://www.redpycs.net/includes /biblio_detalle.php?B_ID=60\&l ang $=1$.

Pizarro, Rodrigo (2007). Hechos y tendencias en frutas y verduras ecológicas. Revista horticultura internacional, 56, 24-27.

Porter, M. (2002). Ventaja competitiva: creación y sostenimiento de un desempeño superior. México: Compañía editorial Continental.

Polonsky, M. J., \& Mintu-Wimsatt, A. (1997). Environmental Marketing: Strategies, Practice, Theory, and Research. Estados Unidos: The Haworth Press Inc.

Proctor, L. (2009, Julio). Costco \& green marketing. Recuperado de http://blog.firefly180.com/blog/ marketing-matters/0/0/costcogreen-marketing

Puro Marketing (2010, septiembre 13). Ikea, nívea, Microsoft, dove entre las marcas más ecológicas. Recuperado de http://www.puromarketing.com/ 53/7933/ikea-nivea-microsoftdove-entre-marcasecologicas.html
Quintero, Camilo y Bautista Germán (2009). Desarrollo de un brochures para la empresa imagen \& marca Ltda., enmarcado dentro de las tendencias del Green marketing para generar innovación en las campañas de comunicación de sus clientes en el segundo semestre de 2009. (Tesis de pregrado), Pontificia Universidad Javeriana, Bogotá. 
Revista Dinero. (2010, Febrero 16). Las 10 clases de consumidores de Bogotá. Recuperado de http://www.dinero.com/negocios -online/industria/10-clasesconsumidoresbogota_68770.aspx

\section{Rodríguez J.P. Slack B. Comtois} Claude. Green logistic (the paradoxes of). Publicado en el libro "The handbook of logistic and Supply chain management. A.M Brewer, K.J Button and D.A. Hensher.2001

Romero. C. Noelia. Integración estratégica de la responsabilidad social en la USC. Avances en el terreno del desarrollo sostenible, la igualdad de oportunidades y la transparencia.

Salgado, Lizbeth. Subirá, María. Beltrán, Luis. (2009) Consumo orgánico y conciencia ambiental de los consumidores. Problemas del desarrollo, revista latinoamericana de economía. 40 (157), pp. 189-199.

\begin{abstract}
Samper, Juan Ramón, Acciones y prácticas de mercadeo verde en empresas colombianas (casos de estudio), revista Borradores de administración No.22, 2009

Senge Peter (2009). El reto de la sostenibilidad o la revolución necesaria. Harvard Deusto Bussines review. 181, 28-33.
\end{abstract}

Sixto, J. y Salgueiro M. (2008, septiembre). Comunicar el marketing verde y re-valorar la marca en las conciencias organizaciones actuales. En XXXI congreso brasilero de ciencias de comunicación. Natal RN: INTERCOM - sociedad brasilera de estudios interdisciplinarios de comunicación.

Stanton, William. Etzel, Michael. Walker, Bruce. (2007). Fundamentos de marketing. Mexico: MC Graw Hill.

Solar Buzz (2009), Market buzz 2009; Annual World solar PV Market Report, (Online), Disponible en: 
http://energytoolbox.org/gcre/bi bliography/65_PVMARKETSol arbuzz

What price (2008).Supermarkets and the environment. Recuperado de http://www.whatprice.co.uk/rec ycling/green-supermarket.html

Tierney, R. (2007). Going green sustainable practice take root in hospitality. HSMAI marketing review. Summer, 24-46.

Trending Marketing (2010, Enero). 10 tendencias de marketing para el 2010.De: Diana Nieto. Recuperado de http://trendymkt.blogcindario.co m/2010/01/00004-10tendencias-de-greenmarketingpara-el-2010.html.

Vazifehdust, Hosain. Javad, Mohammad. Gharib, Zahra (2011). Social marketing, green marketing: the extension of marketing concept. European journal of Economics, Finance and Administrative Sciences.
Vermillion, L. Peart, J. (2010). Green marketing: making sense of the situation. En Allied academies international conference,.(15(1) pp. 68-71). New Orleans: Academy of marketing studies.

Vicente, M. A., Mediano, L. Propuestas para una segmentación estratégica de mercadeo ecológico. Cuadernos de gestión Vol. 2, 1, 11-30.

Vida sana (2010, febrero 18) Marketing ecológico y sus funciones. Recuperado dehttp://www.vidasana.org/notic ias-vidasana/marketingecologico-y-sus-funciones.html

Yánez H. José. (2009). Reformas tributarias y objetivos económicos. Revista Economía y Administración, DiciembreNoviembre, p. 17. ISSN 0764793.

41. PP 20 - 33. 